\section{Europe's cash crisis puts space plans under threat}

Space scientists in Europe are pleading with ministers to significantly boost the European Space Agency's (ESA's) science programme when they meet to decide its budget for the next five years. Without such an increase, researchers fear that high-profile missions may have to be abandoned.

${ }^{\alpha}$ We are struggling to keep control of the budget," says Marcello Coradini, coordinator of Solar System Missions at ESA headquarters in Paris. Costs of science missions in development are predicted to exceed available money by $€ 300$ million (US $\$ 350$ million) to $€ 400$ million over the coming decade.

If money allocated by ESA's ministerial council, which meets in Berlin on 5-6 December, does not alleviate the pressure on the programme's finances, scientists say it may not be possible to cut back missions without undermining their scientific goals. And agency officials say they may be reluctant to absorb the costs - roughly equivalent to one year of spending - through delays, because that means no new missions can be started.

The situation has led to speculation that BepiColombo, a mission destined for a 2013 launch to Mercury, might be cancelled. "That is the big danger painted in the sky," says Karl-Heinz Glassmeier, principal investigator on one of the instruments proposed for the spacecraft.

Nerves were set jangling about the project, which also involves the Japan Aerospace Exploration Agency, after it was postponed because the initial design was too heavy. That problem seems to have been solved, but officials say the estimated cost of the mission, at

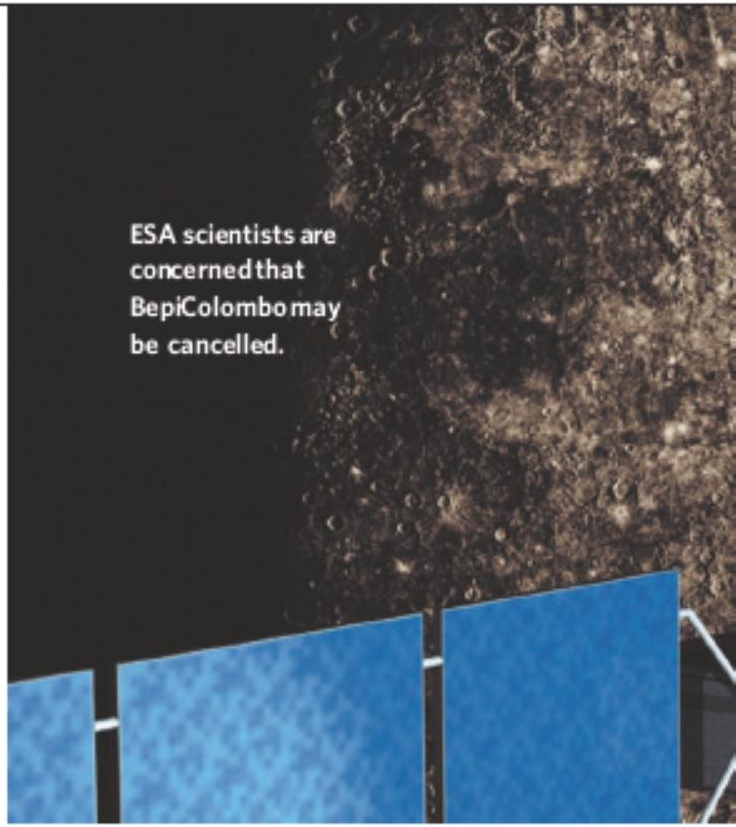

$€ 600$ million to $€ 650$ million, is still more than $€ 100$ million above target.

Researchers on BepiColombo are not the only ones worried. "We're all biting our nails,"

\title{
Hayabusa ready to head home with asteroid sample
}

\section{TOKYO}

Japan's latest space mission seems to have succeeded in its second attempt to collect pieces of a small asteroid. If so, this will be the first time a sample has been collected for return to Ear th from any object in the Solar System apart from the Moon. However, engine trouble casts doubts on whether the craft can returnhome safely.

On25 November, the Japan Aerospace Exploration Agency (JAXA) said data sent from Hayabusa show that allstages of the sampling process went well. Agency engineers said it was almost certain that Hayabusa's sampler had toucheddown on the Itokawa asteroid as planned, and shot two metal pellets into the rock to throwup fragments of the sur face. "I think we collected a sample," said project manager Jun'ichiro Kawaguchi.

Hayabusa was launchedin May 2003, and arrived this September at I tokawa - a potato-shaped, 540metre-long asteroid located about 300 million kilometres from Earth.
Hayabusa is expected toleave I tokawa by early December for its return journey. Whethera sample was definitely collected will not be known until the craftreaches Earth in the summer of 2007.

Toreach this stage, the members of the Hayabusa team have endured a rough time. Two of the craft's three

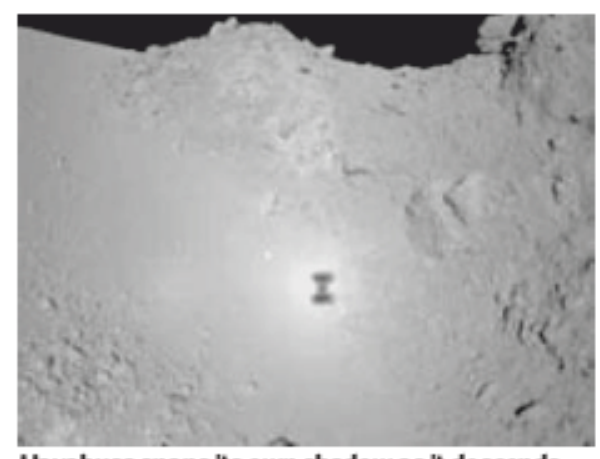

Hayabusa snaps its own shadow as it descends. reaction wheels, which stabilize the probe and help navigation, stopped working; the first failed in July and the second in October. Chemical engines on board were used instead. But their lower accuracy made landing more difficult. In the first landing attempt, on 20 Nowember, Hayabusa seemed to park on the asteroid's hot surface for more than $\mathbf{3 0}$ minutes, and failed to collect a sample.

Time and fuel were running short, so the $\mathbf{2 5}$ November try was almost the last chance to collect a sample. "It was learning in real time," says Donald Yeomans, US project scientist for Hayabusa and senior research scientist at NASA's JetPropulsion Laboratory in Pasadena, California. With each attempt, he says, "they learned more and more about how the spacecraft behaves".

Still, concerns remain. Hayabusa lost its balance soon after departing from the asteroid. Engineers are investigating the cause, butone possibility is that the craft's long stay on Itokawa's hot surface during the first landing attempt damagedone or more of its 12 chemical engines. JAXA said that if the trouble could not be fixed, it would be difficult for Hayabusa to return to Earth.

But astronomers have praised Hayabusa's achievements so far as showing the way for future asteroid missions - especially those involving the operation of ionpropulsion engines and delivery of high-resolutionimages. Analysing the sample, assuming itmakes it back to Ear th, would also help to answer questions about how the Solar System was created.

The mission is renewing Japan's confidence in space activities. JAXA has recently tried a string of high-riskmissions, but has seen many failures over the past few years. "Hayabusa's success has become a tailwind for Japan's space development," Hajime Inoue, JAXA's executive director, said at a press conference. "It proves that the way we have beendoing things wasn'twrong." Ichiko Fuyuno 
